\title{
AITERIDAD Evaluación y coevaluación de aprendizajes en blended learning en educación superior
}

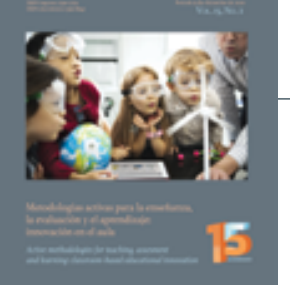

\section{Evaluation and co-evaluation of learning in b-learning in higher education}

D Oscar Maureira-Cabrera es docente e investigador de la Universidad Católica Silva Henríquez (Chile) (omaureir@ucsh.cl) (https://orcid.org/0000-000 1-9208-9708)

(D) Mario Vásquez-Astudillo es docente e investigador de la Universidad Federal de Santa María (Brasil) (mario.astudillo@ufsm.br) (https://orcid.org/0000-000 1-8467-5184)

(1) Francisco Garrido-Valdenegro es docente e investigador del Colegio Salesianos Alameda (Chile) (fgarrido@salesianosalameda.cl) (https://orcid.org/0000-0001-8467-5184)

(1) María José Olivares-Silva es docente e investigadora del Colegio Almenar de las Vizcachas (Chile) (mariajoseolivares@almenar.cl) (https://orcid.org/0000-0002-1775-9262)

Recibido: 2019-10-14 / Revisado: 2020-05-28 / Aceptado: 2020-06-06 / Publicado: 2020-07-01

\section{Resumen}

El estudio evalúa la utilización de herramientas tecnológicas para implementar evaluación y coevaluación realizada por los estudiantes, en función del aprendizaje autónomo y la evaluación por parte del profesor, aprovechando el potencial de la retroalimentación instantánea de las herramientas que disponen los Ambientes Virtuales de Aprendizaje. La extensa implementación de estos ambientes virtuales en la educación superior hace que el blended learning sea una nueva normalidad, automatizando y facilitando el monitoreo de los avances en el aprendizaje, a través de la gestión pedagógica de prácticas efectivas. La propuesta de innovación propende al desarrollo de la autonomía y protagonismo de estudiantes en el logro de sus aprendizajes. El diseño metodológico es correlacional que busca establecer la asociación entre la confección de preguntas y resolución de test online por parte de los estudiantes y su nivel de desempeño en pruebas de conocimiento administradas por el profesor. Se aplicó en seis grupos de las cohortes 2016 y 2017 en la asignatura Investigación Educativa para carreras de pedagogías de una universidad chilena. Los principales resultados apuntan a una significativa correlación entre uso del ambiente virtual en la construcción y aplicación de instrumento tipo test y los resultados de desempeño en pruebas de conocimiento. Se concluye que el aprendizaje de los alumnos se potencia, al integrar la evaluación como parte del proceso formativo con un rol activo y autónomo de los estudiantes con apoyo de tecnologías.

Descriptores: Autonomía, evaluación online, coevaluación, aprendizaje, b-learning, educación superior.

\begin{abstract}
The study examines the use of technological tools to implement self and peer-assessment based on students' autonomous learning and formal evaluation carried out by the teacher, taking advantage of the potential of instant feedback from the tools available in Virtual Learning Environments (VLE). The extensive implementation of VLE in higher education makes blended learning an emergent modality, automating and facilitating the monitoring of learning progress, through the pedagogical management of effective practices. The innovation proposal aims at fostering students' autonomy and their central role in the achievement of their learning. The methodological design is correlational seeking to establish the association between the preparation of questions and online test resolution by the students and their level in performance tests administered by the teacher. It was carried out in six groups of the 2016 and 2017 cohorts in the Educational Research subject within initial teacher training programs at a Chilean university. The main
\end{abstract}

Forma sugerida de citar: Maureira-Cabrera, O., Vásquez-Astudillo, M., Garrido-Valdenegro F., \& Olivares-Silva, M.J. (2020). Evaluación y coevaluación de aprendizajes en blended learning en educación superior. Alteridad, 15(2), 190 -203. https:// doi.org/10.17163/alt.v15n2.2020.04 
results show a significant correlation between the use of the virtual environment in the construction and application of a self and peer assessment instruments in a test- typed format and their results in performance tests. It is concluded that student learning is enhanced by integrating assessment as part of the teacher training process with an active and autonomous role of students supported by educational technologies.

Keywords: Autonomy, online evaluation, coevaluation, learning, blended learning, higher education.

\section{Introducción}

La informática emerge a mediados del siglo XX formada por un conjunto de tecnologías encargadas del almacenamiento y automatización de la información que, con el crecimiento tecnológico moderno, penetró en diversos ámbitos del hacer social, en particular, los centros educacionales básicos, medios y universitarios. En la actualidad, la educación superior ha incorporado Tecnologías de la Información y Comunicación (TIC) en los procesos de enseñanza, con la finalidad de apoyar los procesos de enseñanza-aprendizaje de alumnos y docentes (Luna et al., 2018). Esto genera demandas de nuevas estrategias didácticas que afronten dicho encargo, incorporando estrategias que incluyan las nuevas tecnologías (Bahamondes \& Ponce, 2012).

La innovación en los sistemas educativos se ha convertido en una necesidad imperiosa en la educación superior, en tanto busca cambios y mayor difusión de las experiencias exitosas, ya que la universidad necesita cambiar en todos sus niveles para sobrevivir y prosperar en el nuevo "mercado educativo" (Quesada et al., 2017). Para el profesorado, esto significa un consumo importante de recursos y tiempo, lo que hace necesario, entre otras cuestiones, analizar su eficacia en el resultado académico y la calidad de la enseñanza a través de la aplicación de estas nuevas técnicas (Vivel-Bua, et al., 2015).

La integración y uso intensivo de tecnologías digitales, en particular basadas en la web están transformando las universidades en todo el mundo (Duart \& Mengual, 2015; Boelens et al., 2018; Dziuban, et al., 2018). Las institucio- nes de educación superior han incorporado las plataformas tecnológicas ricas en medios para la evaluación, así como los "cursos personalizados o adaptativos y las herramientas de conferencia web, capaces de conectar a los estudiantes para actividades a distancia sincrónicas, se están convirtiendo en soluciones comunes para los diseños de aprendizaje blended learning (BL)" (Alexander et al., 2019, p. 12), pues la tecnología tiene el potencial de aumentar el acceso a la educación, mejorar las experiencias de aprendizaje y reducir el costo de proporcionar educación postsecundaria de alta calidad (Protopsaltis \& Baum, 2019).

\subsection{Modalidad b-learning}

La modalidad b-learning se está constituyendo en la nueva normalidad, dada su extensiva adopción en la educación superior como efecto de la integración de tecnologías las que posibilitan nuevos espacios de aprendizaje, nuevas estrategias de enseñanza y evaluación por parte de los profesores. Diversos autores ofrecen distintas definiciones con énfasis ya sea en la tecnología y como esta es usada en las instancias presencial y on ine, o en las estrategias o en procesos pedagógicos, con lo cual Picciano et al. (2014) constatan que no existe un consenso sobre una definición, sin embargo existe un elemento en común, tal como ya lo constatara Vaughan (2010, p. 23), que es "combinación o integración de la modalidad de educación presencial y no presencial sustentada en las Tecnologías de la Información y Comunicación (TIC)". Por tanto, como concluyen Roza et al. (2019), el BL no excluye una modalidad educativa en función de la otra, al contrario, propone usar más y mejor el potencial disponible en cada modalidad para que el aprendizaje significativo ocurra a través de prácticas efectivas.

En cuanto a la efectividad del BL, se aprecian buenos resultados académicos en términos de menores tasas de abandono (López et al., 2013) y mayor rendimiento académico, con diferentes grados de significatividad estadística de la modalidad $\mathrm{BL}$, respecto a la presencial tradicional (Carranza \& Caldera, 2018; Essam, 2010; 
Halverson \& Graham, 2019; Li et al., 2014; López et al., 2013; Rienties \& Toetenel, 2016); aunque la percepción positiva de los estudiantes hacia los cursos BL no siempre refleja una mejora en los resultados de aprendizaje (Sajid et al., 2016).

A partir de la irrupción de la revolución del BL, se generan nuevas posibilidades de interactividad desde la perspectiva didáctica, en la que "las nuevas formas de enseñar y aprender han de ser diseñadas, estudiadas y comprendidas en sus interacciones con los nuevos medios y contextos de aprendizaje" (Bartolomé et al., 2018, p. 35). Estos nuevos contextos y ambientes de aprendizaje generan comunidades de aprendizaje que facilitan y sostiene el aprendizaje mientras promueven la interacción, la colaboración y la construcción de un sentido de pertenencia entre los miembros, elementos clave del éxito del BL, que debe darse en un proceso dinámico, flexible y adaptativo.

\subsection{Aula virtual}

Entre las herramientas informáticas utilizadas en los procesos educativos se hallan las aulas virtuales, cuya mayor ventaja en un modelo presencial de enseñanza es que libera al profesor y al alumno de la coincidencia temporal y espacial propia de la enseñanza tradicional al flexibilizar itinerarios personales de desarrollo de capacidades procesual, exploratoria y visualización (Barberà \& Badia, 2005). Así, se interactúa en un lapsus de tiempo y espacio donde el aprendiz es capaz de trabajar autónomamente según su propio ritmo y tiempos de aprendizaje. Esta plataforma representa una atractiva herramienta pedagógica que opera en modalidad e-learning como b-learning.

El uso de las TIC introdujo cambios en el sistema educativo, por ejemplo, en el aprendizaje a distancia (Cardona \& Sánchez, 2010), dado el surgimiento de Ambientes Virtuales de Aprendizaje (AVA) o Espacios Virtuales de Aprendizajes (EVA) sostenidos por la plataforma Learning Management System (LMS). El LMS es un tipo de software destinado al servicio pedagógico y designado a administrar contenidos y actividades formativas de una específica organización (Arias \& Venegas, 2013), los cuales son distribuidos en aulas virtuales (un EVA) o especies de aula de estudio tradicional, cuya diferencia es el canal de comunicación que puede ser sincrónica y/o asincrónica.

En el aula virtual puede haber o no haber coincidencia temporal, es decir, aprendizaje sincrónico o asincrónico. Según el contexto virtual, la sincronía se traduce en talleres, seminarios o debates que requieren la presencia coincidente en el tiempo, aunque distante, entre profesor y aprendiz, mientras que en la asincronía estas pueden no coincidir en tiempo y espacio (Rojas et al., 2014), permitiendo que el rol pasivo del alumno frente al aprendizaje se convierta en activo y los docentes pasen de un modelo clásico a uno facilitador de aprendizajes (Careaga \& Fuentes, 2012).

El aula virtual debe concebirse como una herramienta social y educativa debido a sus funciones de socializador, informativo, formativo y formador, motivador, evaluador, comunicativo, organizador, analítico, innovador e investigador (Barberá \& Badia, 2005), que dan sentido a su inclusión en los centros educativos. Asimismo, facilita el perfil e-learning (desarrollo de la formación a distancia), pues ha generado que el modelo b-learning (aprendizaje semipresencial), propio de los programas de pregrado cuyo formato dicotómico combina clases presenciales y actividades de forma e-learning, lo incorpore en los procedimientos al promover un diseño metodológico colaborativo.

En esta perspectiva, García-Beltrán et al. (2016) establecen que las principales ventajas de usar entornos virtuales para la coevaluación, por medio de pruebas objetivas, yacen en el seguimiento individualizado del aprendizaje del alumno, la facilitación del establecimiento de una evaluación continua en el proceso de aprendizaje, la evaluación de conocimientos y habilidades, la reducción del tiempo de diseño, distribución y desarrollo, aportan "una gran flexibilidad temporal y espacial del sistema 
tanto para la configuración de ejercicios como de su realización. En este sentido puede ser especialmente útil para permitir que el alumno pueda seguir su propio ritmo de aprendizaje" (2016, p. 4).

\subsection{Pruebas objetivas en espacios virtuales}

Las pruebas de respuesta objetiva en un entorno virtual cuentan con la singularidad de ser implementadas de modo sencillo y tener corrección y retroalimentación automática. Estas, también, pueden ser usadas para motivar y orientar a los alumnos en el proceso de formación e, incluso, como medio de autoevaluación de cada lección o contenido de una asignatura (García-Beltrán et al., 2016). Según Pacheco et al. (2014) el empleo del aula virtual como herramienta para la evaluación de contenidos permite un seguimiento a los procesos formativos y el aprendizaje grupal a través de actividades colaborativas, además de garantizar el diálogo profesor-estudiante y favorecer el seguimiento continuo del avance académico, lo que exige al alumno una preparación permanente.

En particular, el aula virtual de la Universidad Católica Silva Henríquez (UCSH) cuenta con la actividad cuestionario (sistema de pruebas de respuesta objetiva) que permite al docente diseñar y proponer exámenes con preguntas de opción múltiple, verdadero/falso, coincidencia, respuesta corta y respuesta numérica. Sobre la configuración de esta actividad, el profesor puede regular la cantidad de intentos permitidos, establecer un tiempo límite, formular el test con preguntas ordenadas o seleccionadas del banco de preguntas, calificar sumativa y automáticamente cada intento en el libro de calificaciones (excepto las preguntas tipo ensayo) y determinar cuándo se muestran los resultados, la retroalimentación y las respuestas correctas (UCSH, 2016). La retroalimentación inmediata de las coevaluaciones en entornos virtuales es fundamental en el proceso de aprendizaje como elemento motivador y orientador para el estudiante (García-Beltrán et al., 2016).

\subsection{Las preguntas desde la autonomía}

El concepto de autonomía tiene una holgada definición y es un ejemplo característico de la diversidad semántica que toman los términos dentro del campo de la educación. La autonomía se asocia a la capacidad de tomar decisiones acordes a criterios propios y se contrapone a la "heteronomía", que significa ejecutar las decisiones emanadas de los demás (Sarramona, 2011). Para Kamii y López (1982) la autonomía es la capacidad de pensar con originalidad y sentido crítico desde varios puntos de vista, donde los intercambios de información y negociaciones con pares son importantes en el desarrollo intelectual. El estudiante autónomo logra un aprendizaje duradero, continuo y una actitud hacia el saber, desde una enseñanza colaborativa, significativa, sincrónica y asincrónica que exige una metodología para desarrollar en el aprendiz habilidades de autoformación, asociación del estudio con la formulación de preguntas y respuestas, búsqueda de información y fomento de la mentalidad crítica y creativa (Massié, 2010).

La autonomía, entonces, debe ser entendida como el fin último de la educación, la cual representa aprender a aprender, facultad propia de una persona que dirige, regula y evalúa conscientemente su formación (Manrique, 2004). Según Castillo et al. (2006) aprender a aprender implica una capacidad reflexiva sobre los medios que generan un aprendizaje, competencia con la cual se elaboran estrategias flexibles y calificadas que garantizan el dominio de adecuación. Las estrategias de aprendizajes son un conjunto de métodos que favorecen el estudio, de las cuales destacan:

- Previas a la lectura: Establecen el propósito de la lectura. En específico, activan conocimientos previos y elaboran predicciones o preguntas.

- Durante la lectura: Se aplican mientras se interactúa directamente con el texto, por 
ejemplo, subrayar una frase importante, crear notas o elaborar conceptos.

- Posteriores a la lectura: Ocurren una vez finalizada la lectura, las más típicas son resúmenes, definir ideas principales y formular preguntas con sus respuestas.

La formulación de una buena pregunta manifiesta en las personas un proceso positivo de aprendizaje, porque la capacidad de diseñar buenas preguntas es una competencia esencial para desarrollar un pensamiento crítico (López, 2011). Dicha habilidad es clave en los procedimientos destinados a comprender un texto, su utilización respalda el logro de alcanzar niveles más profundos de comprensión, pues la formulación de preguntas exige al lector tener conciencia de los procesos de comprensión, o sea, realizar una adecuada interrogante implica tener la capacidad de evaluar la propia intelección y generar conocimiento determinado para la formulación precisa de una pregunta (Silvestri, 2006). Para Morón (2015) la formulación de buenas preguntas en ocasiones es más importante que las respuestas debido al proceso cognitivo que se produce, adecuado para la adquisición de la competencia de aprender a aprender.

\subsection{Evaluación desde la teoría constructivista}

En los últimos años la nueva cultura de consumo, las demandas sociales y la necesidad de rendir cuentas a la administración educacional han producido cambios en el enfoque de la valoración de aprendizajes (Parra, 2008). Esto ha convertido a la evaluación en un fenómeno que provoca en los alumnos ansiedad y pensamientos que incitan conflictos académicos (Bausela, 2005). No obstante, Alfaro (2000) señala que en un contexto donde los estudiantes son medidos desde un paradigma constructivista la evaluación es una herramienta fundamental, compleja $y$ relevante en el quehacer docente. Bajo estos términos, la evaluación debe estar y ser perma- nente en el hacer pedagógico, además de promover la posibilidad de generar nuevo aprendizaje en el educando, es decir, los instrumentos de evaluación no solo son medios de valoración de conocimientos, sino también formas de enseñanza (Parra, 2008).

Ahora bien, la evaluación como elemento educativo tiene carácter político, por eso, se puede abordar desde perspectivas diferentes como la conservadora (el docente tiene el poder absoluto) o progresista (el poder se distribuye entre profesor y estudiante) según Quesada et al. (2017). En materia de didáctica, se exige fomentar la participación de los alumnos en su evaluación a través de la autoevaluación y coevaluación, para que desarrollen su autonomía y capacidad de aprender a aprender (González et al. 2007) al colaborar con el profesor en la tarea de evaluar.

En este sentido, la coevaluación para los docentes en formación figura una oportunidad para desarrollar la capacidad de crear instrumentos confiables de evaluación dentro de sus competencias adquiridas como se plasma en el estándar pedagógico 6: "[el docente] conoce y sabe aplicar métodos de evaluación para observar el progreso de los estudiantes y sabe usar los resultados para retroalimentar el aprendizaje y la práctica pedagógica" (Ministerio de Educación de Chile, 2012, p. 43). La coevaluación se define como la instancia en que un alumno mide el logro de los aprendizajes de sus pares (Parra, 2008) y es parte de la evaluación formativa en el proceso de enseñanza-aprendizaje, pues deja conocer, regular y mejorar el aprendizaje del sujeto (González et al., 2007). Este proceso cuenta con tres características importantes (Alvares, 2008):

- Permite al alumno evaluar conocimientos, tradicionalmente atribuido al docente.

- Desarrolla habilidades para el proceso de evaluación y diseño de instrumentos de medición.

- El estudiante autoevalúa sus conocimientos.

Una de las técnicas que han tenido mayor difusión en el ámbito pedagógico evaluativo es 
el One Minute Paper, esto es, preguntas que los alumnos deben resolver por escrito al finalizar una sesión de clase y que posterior a su revisión el profesor expone los resultados obtenidos enfocándose en los errores o deficiencias. Esta técnica puede incorporar la identificación de conceptos clave, formulación de ejemplos, redacción de ideas y opiniones sobre el tema abordado, etc. Ahora bien, el tiempo que demanda la revisión de estos cuestionarios es excesiva, por lo que se presenta como uno de sus inconvenientes, de ahí que se recomiende un muestreo aleatorio o el uso de las nuevas tecnologías (Vivel-Bua et al., 2015).

\section{Metodología}

\subsection{Participantes}

Los participantes del estudio son estudiantes de una muestra de 6 grupos de la asignatura Investigación Educativa del primer y segundo semestre, la cual se ubica en el penúltimo año de formación inicial docente, previa al seminario de grado de licenciatura en educación y práctica profesional del Programa de Formación Pedagógica General, común a todas las carreras de la formación inicial del profesorado de la UCSH.

\subsection{Pregunta e hipótesis de investigación}

La pregunta de investigación planteada es ¿existe una asociación entre el uso del aula virtual, por medio de la valoración en la construcción y aplicación de un instrumento coevaluativo en formato tipo test diseñados por estudiantes y el desempeño en pruebas de conocimiento afines a conceptos clave de investigación educativa?

Basándonos en el referencial teórico, la hipótesis de trabajo definida es que existe una asociación positiva y estadísticamente significativa entre la calificación de preguntas construida por estudiantes, un test que agrupa a éstas desarrollada en aula virtual y el desempeño en una prueba de conocimiento.
El enfoque adoptado es cuantitativo con un diseño no experimental transeccional y de alcance descriptivo correlacional. De modo que, por medio de indicadores cuantitativos agregados al uso del aula virtual (valoración en construcción de preguntas y resolución de test afines), se buscó relacionar tal intensidad de actividad con el nivel de desempeño de estudiantes en ítems de pruebas de conocimiento afines a conceptos clave de investigación. Las variables delimitadas son uso del aula virtual (V1) y desempeño en pruebas de conocimiento (V2).

\subsection{Descripción de las variables}

Variable 1 (V1) uso del aula virtual: Corresponde a la valoración del uso del aula virtual en la construcción y aplicación de un instrumento coevaluativo en formato tipo test, diseñados por estudiantes de la asignatura Investigación Educativa. Los valores de la V1 se obtienen del promedio entre la subvariable valoración de elaboración cuestionario (V1.1) y la subvariable calificación respuesta cuestionario online (V1.2).

La subvariable V1.1 está referida a la valoración objetiva sobre la calidad de las preguntas de selección múltiple generadas por alumnos, con la justificación de la alternativa correcta y calificada por un experto en una escala de 1 a 7 . La subvariable V1.2 es la calificación obtenida por la muestra de estudiantes en cada cuestionario online formulados con una selección de preguntas sobre el total con mayor valoración de la subvariable V1.1.

La subvariable V1.2 se obtiene al promediar las calificaciones (escala de 1 a 7 con exigencia del 60\%) obtenidas por los estudiantes en la resolución de cuestionarios online de cada periodo. Cada cuestionario cuenta con la posibilidad de usar dos intentos, donde el alumno probará y valorará sus conocimientos a partir de la calificación obtenida en el primer ensayo, además de recibir una retroalimentación con las respuestas correctas. Estos cuestionarios incluyen preguntas seleccionadas de la subvariable V1.1 entre aquellas cuyo nivel de logro es el más alto. El com- 
portamiento de las preguntas y respuestas de los cuestionarios es de orden aleatorio para que el aprendiz no memorice las alternativas, más bien recuerde la pregunta y respuesta correcta a fin de promover el aprendizaje de los conceptos clave desde la autoevaluación y coevaluación.

Variable 2 (V2) dominio de conocimientos: Corresponde a los resultados de desempeño en pruebas de conocimiento en la calificación (escala de 1 a 7) obtenida por los alumnos en el ítem de selección múltiple perteneciente a la primera prueba de conocimientos sobre conceptos básicos de la asignatura Investigación Educativa del semestre cursado con exigencia del $60 \%$ sobre un total de 16 preguntas.

\subsection{Descripción de las variables}

La variable V1 corresponde al promedio entre dos subvariables denominadas V1.1 e V1.2. La variable V1.1 (valoración de elaboración cuestionario) es una valoración objetiva sobre la calidad de las preguntas de selección múltiple generadas por alumnos, con la justificación de la alternativa correcta y calificada por un experto. La variable V1.2 (calificación respuesta cuestionario online) es la calificación obtenida por la muestra de estudiantes en cada cuestionario online formulados con una selección de preguntas sobre el total con mayor valoración de la variable V1.1.

La variable V1.1 se obtuvo al promediar las calificaciones (escala de 1 a 7 ) obtenidas por los alumnos en las etapas de desarrollo de preguntas de comprensión del texto "Metodología de la investigación" (Hernández et al., 2012) e "Investigación educativa" (McMillan \& Schumacher, 2005).

La variable V1.2 se obtiene al promediar las calificaciones (escala de 1 a 7 con exigencia del $60 \%$ ) obtenidas por los estudiantes en la resolución de cuestionarios online de cada periodo. Cada cuestionario cuenta con la posibilidad de usar dos intentos, donde el alumno probará y valorará sus conocimientos a partir de la calificación obtenida en el primer ensayo, además de recibir una retroalimentación con las respuestas correctas. Estos cuestionarios incluyen preguntas seleccionadas de la variable V1.1 entre aquellas cuyo nivel de logro es el más alto. El comportamiento de las preguntas y respuestas de los cuestionarios es de orden aleatorio para que el aprendiz no memorice las alternativas, más bien recuerde la pregunta y respuesta correcta a fin de promover el aprendizaje de los conceptos clave desde la autoevaluación y coevaluación. La variable V2 corresponde a la calificación (escala de 1 a 7) obtenida por los alumnos en el ítem de selección múltiple perteneciente a la primera prueba de conocimientos sobre conceptos básicos de Investigación Educativa del semestre cursado con exigencia del $60 \%$ sobre un total de 16 preguntas.

\subsection{Instrumentos}

La valoración objetiva en la construcción de preguntas sobre la literatura obligatoria (V1.1) yace en el criterio de un experto, quien basa su análisis en las reglas de construcción de preguntas de selección múltiple de Parra (2008), dada la intención de mantener distancia y asegurar la objetividad en el proceso, así como garantizar el correcto contenido de las preguntas. Para medir el aprendizaje mediante el uso de los cuestionarios online desde la coevaluación de contenidos (V1.2) se utiliza una escala de 1 a 7 con exigencia del $60 \%$ sobre el total de preguntas. Los cuestionarios fueron construidos con preguntas seleccionadas de la variable V1.1 entre aquellas cuyo nivel de logro fuese valor 7,0.

La herramienta virtual permite: Ordenar aleatoriamente tanto las preguntas del test en cada intento como las alternativas de cada pregunta y configurar la visualización de las estas, entregar los resultados y retroalimentación de forma instantánea, limitar el tiempo de resolución de las preguntas y resolver o responder desde cualquier lugar del mundo.

\section{Resultados}

A continuación, se describe en una sola tabla agregada una síntesis de los resultados obtenidos en las distintas categorías: 
Tabla 1. Estadísticos

\begin{tabular}{|c|c|c|c|c|c|}
\hline \multicolumn{6}{|c|}{ Estadísticos V1.1 y V1.2 2016(2) } \\
\hline \multicolumn{3}{|c|}{ V1.1 } & \multicolumn{3}{|c|}{ V1.2 } \\
\hline \multirow[t]{2}{*}{$\mathrm{N}$} & Válidos & 47 & $\mathrm{~N}$ & Válidos & 47 \\
\hline & Perdidos & 0 & & Perdidos & 0 \\
\hline Media & & 3,2 & & & 4,3 \\
\hline Mediana & & 2,7 & & & 5,2 \\
\hline Moda & & 1,8 & & & 1 \\
\hline $\begin{array}{l}\text { Desviación } \\
\text { estándar }\end{array}$ & & 1,96 & & & 2,11 \\
\hline Varianza & & 3,8 & & & 4,46 \\
\hline C.V & & $61,25 \%$ & & & $49,06 \%$ \\
\hline \multicolumn{6}{|c|}{ Estadísticos V1.1 y V1.2 2017(1A) } \\
\hline \multicolumn{3}{|c|}{ V1.1 } & \multicolumn{3}{|c|}{ V1.2 } \\
\hline $\mathrm{N}$ & Válidos & 115 & $\mathrm{~N}$ & Válidos & 115 \\
\hline & Perdidos & 0 & & Perdidos & 0 \\
\hline Media & & 3,8 & & & 4,4 \\
\hline Mediana & & 4 & & & 5,3 \\
\hline Moda & & 1 & & & 1 \\
\hline $\begin{array}{l}\text { Desviación } \\
\text { estándar }\end{array}$ & & 2,1 & & & 2,1 \\
\hline Varianza & & 4,4 & & & 4,4 \\
\hline C.V & & $55,26 \%$ & & & $47,72 \%$ \\
\hline
\end{tabular}

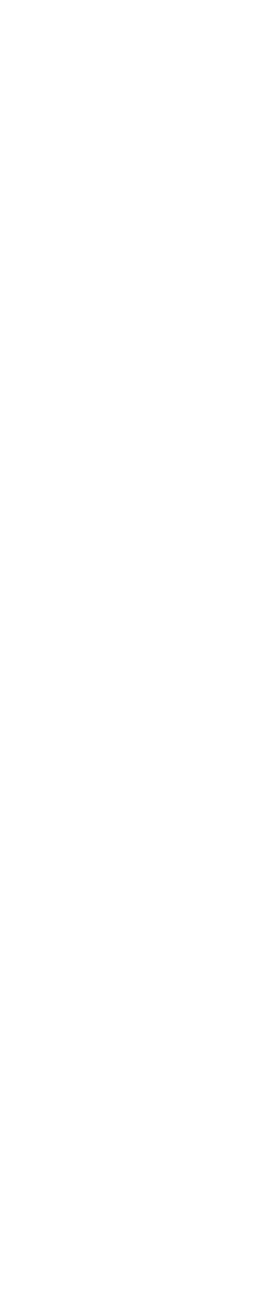

Estadísticos V1.1 y V1.2 2017(1B)

V1.1

\begin{tabular}{|l|l|c|l|c|c|}
\hline N & Válidos & 47 & N & Válidos & 47 \\
\hline Media & Perdidos & 0 & & Perdidos & 0 \\
\hline Mediana & & 3,9 & & & 4,9 \\
\hline Moda & & 4 & & & \\
\hline $\begin{array}{l}\text { Desviación } \\
\text { estándar }\end{array}$ & & 2,23 & & 2,18 \\
\hline Varianza & & 4,96 & & & 4,76 \\
\hline C.V. & & $57,17 \%$ & & & $44,49 \%$ \\
\hline
\end{tabular}

\begin{tabular}{|l|l|c|l|l|c|}
\hline \multicolumn{5}{|c|}{ V1 } & \multicolumn{1}{c|}{ V2 } \\
\hline N & Válidos & 47 & N & Válidos & 43 \\
\hline & Perdidos & 0 & & Perdidos & 4 \\
\hline Media & & 3,75 & & & 4,2 \\
\hline Mediana & & 3,8 & & & 4,4 \\
\hline Moda & & 1,4 & & & 4,4 \\
\hline Desviación & & 1,72 & & & 1,65 \\
\hline Varianza & & 2,99 & & & 2,73 \\
\hline C.V. & & $45,86 \%$ & & & $39,3 \%$ \\
\hline
\end{tabular}




\begin{tabular}{|c|c|c|c|c|c|}
\hline \multicolumn{6}{|c|}{ Estadísticos V1 y V2 2017(1A) } \\
\hline \multicolumn{3}{|c|}{ V1 } & \multicolumn{3}{|c|}{ V2 } \\
\hline \multirow[t]{2}{*}{$\mathrm{N}$} & Válidos & 115 & $\mathrm{~N}$ & Válidos & 95 \\
\hline & Perdidos & 0 & & Perdidos & 20 \\
\hline Media & & 4,15 & & & 3,86 \\
\hline Mediana & & 4,6 & & & 3,8 \\
\hline Moda & & 1 & & & 3,2 \\
\hline Desviación & & 1,78 & & & 1,07 \\
\hline Varianza & & 3,16 & & & 1,14 \\
\hline C.V. & & $42,89 \%$ & & & $27,72 \%$ \\
\hline \multicolumn{6}{|c|}{ Estadísticos V1 y V2 2017(1A) } \\
\hline \multicolumn{3}{|c|}{ V1 } & \multicolumn{3}{|c|}{ V2 } \\
\hline $\mathrm{N}$ & Válidos & 114 & $\mathrm{~N}$ & Válidos & 91 \\
\hline & Perdidos & 1 & & Perdidos & 24 \\
\hline Media & & 4,37 & & & 4,07 \\
\hline Mediana & & 4,85 & & & 3,8 \\
\hline Moda & & 1 & & & 2,9 \\
\hline Desviación & & 1,99 & & & 1,14 \\
\hline Varianza & & 3,97 & & & 1,29 \\
\hline C.V. & & $45,53 \%$ & & & $28 \%$ \\
\hline
\end{tabular}

Claves: V1: Uso del aula virtual; V2: Dominio de conocimientos; V1.1: Calidad de las preguntas; V1.2: Calificación cuestionario online; 2016(2): Alumnos de segundo semestre de 2016; 2017 (1A): Alumnos primer semestre de 2017, grupo A, 2017 (1B): grupo B

\subsection{Análisis correlacional}

La relación entre las variables $\mathrm{V} 1, \mathrm{~V} 1.1, \mathrm{~V} 1.2$ y $\mathrm{V} 2$, como V1 es el resultado de promediar V1.1 y V1.2 no aplica buscar una relación entre ellas y una correlación entre V1.1 y V1.2 no tiene relevancia frente a la problemática estudiada, por esto, las relaciones indagadas son V1.1-V2, V1.2-V2 y V1-V2.

Para medir el grado de relación entre las variables se usó el coeficiente de correlación de Pearson, porque estas variables son cuantitativas continuas y de naturaleza lineal. Además, para probar que en realidad están relacionadas y no son producto del azar se aplicó un test de hipótesis estadístico que permite encontrar la significación de dicho coeficiente, es decir, preguntarse por la probabilidad de que tal coeficiente derive de una población cuyo valor sea cero. $\mathrm{Al}$ respecto, se tienen dos hipótesis posibles:

El coeficiente de correlación $H_{1}: x_{r y}=0 \rightarrow$ obtenido procede de una población con correlación cero $(\mathrm{p}=0)$.

El coeficiente de correlación obtenido procede de una población cuya correlación es mayor de cero $(\mathrm{p}>0)$ y se espera probar que se relacionan positivamente. 


\subsection{Correlación V1.1: Calidad de las preguntas; V2: Dominio de conocimientos}

Tabla 2. Correlación V1.1-V2

\begin{tabular}{|c|c|c|c|}
\hline \multicolumn{4}{|c|}{ Correlación V1.1-V2 segundo semestre 2016} \\
\hline \multicolumn{4}{|c|}{ Correlación } \\
\hline \multicolumn{3}{|c|}{ Segundo semestre 2016} & \multirow{3}{*}{$\begin{array}{c}\mathrm{V} 2 \\
0,185 \\
0,235\end{array}$} \\
\hline \multirow{2}{*}{\multicolumn{2}{|c|}{ V1.1 }} & Coef. Pearson & \\
\hline & & Prueba t & \\
\hline \multicolumn{4}{|c|}{ Correlación V1.1-V2 primer semestre 2017 (A) } \\
\hline \multicolumn{4}{|c|}{ Correlación } \\
\hline \multicolumn{3}{|c|}{ primer semestre $2017 \mathrm{~A}$} & V2 \\
\hline \multirow{2}{*}{\multicolumn{2}{|c|}{ V1.1 }} & Coef. Pearson & 0,278 \\
\hline & & Pruebat & 0,006 \\
\hline \multicolumn{4}{|c|}{ Correlación V1.1-V2 primer semestre 2017 (B) } \\
\hline \multicolumn{4}{|c|}{ Correlación } \\
\hline \multicolumn{3}{|c|}{ Primer semestre 2017 B } & V2 \\
\hline \multirow{2}{*}{ V1.1 } & \multirow{2}{*}{\multicolumn{2}{|c|}{$\begin{array}{l}\text { Coef. Pearson } \\
\text { Prueba } t\end{array}$}} & $-0,002$ \\
\hline & & & 0,985 \\
\hline
\end{tabular}

\subsection{Correlación V1.2: Calificación cuestionario online; V2: Dominio de conocimientos}

Tabla 3. Correlación V1.2-V2

\begin{tabular}{|c|c|c|}
\hline \multicolumn{3}{|c|}{ Correlación V1.2-V2 segundo semestre 2016} \\
\hline \multicolumn{3}{|c|}{ Correlación } \\
\hline \multicolumn{2}{|c|}{ Segundo semestre 2016} & \multirow{3}{*}{$\begin{array}{c}\text { V2 } \\
0,457 \\
0,002\end{array}$} \\
\hline \multirow{2}{*}{ V1.2 } & Coef. Pearson & \\
\hline & Prueba $\mathrm{t}$ & \\
\hline \multicolumn{3}{|c|}{ Correlación V1.2-V2 primer semestre 2017 (A) } \\
\hline \multicolumn{3}{|c|}{ Correlación } \\
\hline \multicolumn{2}{|c|}{ Primer semestre $2017 \mathrm{~A}$} & \multirow{3}{*}{$\begin{array}{c}\text { V2 } \\
0,054 \\
0,601\end{array}$} \\
\hline \multirow{2}{*}{ V1.2 } & Coef. Pearson & \\
\hline & Prueba t & \\
\hline \multicolumn{3}{|c|}{ Correlación V1.2-V2 primer semestre 2017 (B) } \\
\hline \multicolumn{3}{|c|}{ Correlación } \\
\hline \multicolumn{2}{|c|}{ Primer semestre 2017 B } & \multirow{3}{*}{$\begin{array}{c}\text { V2 } \\
-0,046 \\
0,659\end{array}$} \\
\hline$V_{11}$ & Coef. Pearson & \\
\hline v1.1 & Pruebat & \\
\hline
\end{tabular}


En el caso de las variables V1.2-V2 segundo semestre 2016 la correlación es 0,457 , es decir, positiva moderada. Sobre la significación, con un 95\% de confianza, se rechaza la hipótesis nula, pues el resultado de la prueba $t$ es menor que 0,05 . Por tanto, V1.2 se relaciona moderada y positivamente respecto a $\mathrm{V} 2$.

Para las variables V1.2-V2 primer semestre 2017 (A) La correlación es 0,054 positiva muy baja. Sobre la significación, con un $95 \%$ de confianza, no se rechaza la hipótesis nula, pues el resultado de la prueba $t$ es mayor que 0,05 . Por ello, los datos estadísticos no son suficientes para asegurar una relación.

En cuanto a las variables V1.2-V2 primer semestre 2017 (B) la correlación es -0,046 negativa muy baja. Respecto a la significación, con un $95 \%$ de confianza, no se rechaza la hipótesis nula, ya que el resultado de la prueba t es mayor que 0,05 . Por ende, los datos estadísticos no son suficientes para asegurar una relación entre estas variables.

\subsection{Correlación V1 Uso del aula virtual; V2 Dominio de conocimientos}

\section{Tabla 4. Correlación V1-V2}

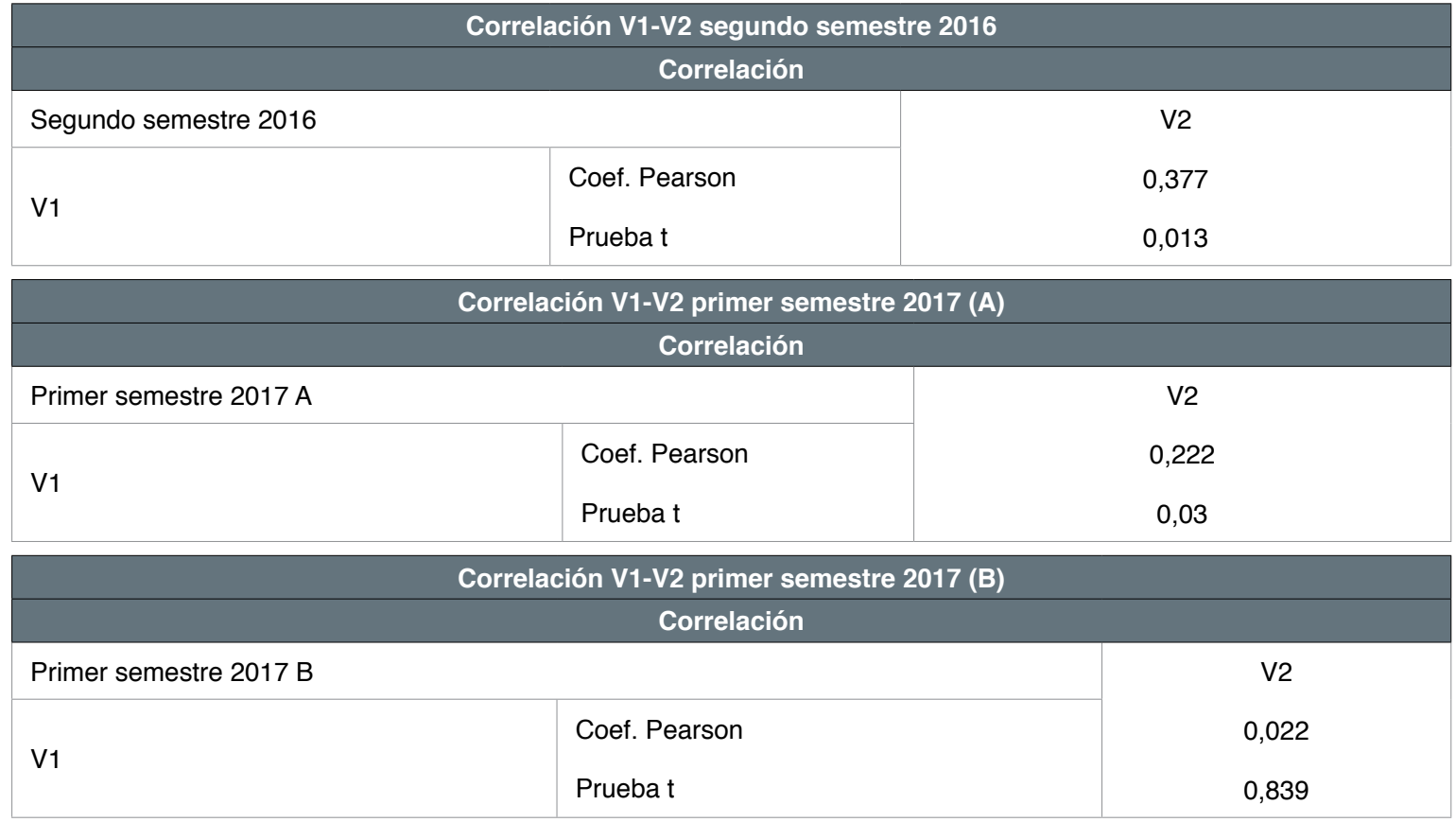

En síntesis, en los dos primeros casos existe una correlación positiva, moderada o baja entre el uso del aula virtual (V1) y el dominio de conocimientos(V2), que es estadísticamente significativa con $95 \%$ de confianza, confirmándose la hipótesis de trabajo. En cambio, en el tercer caso, tal situación no se da.

\section{Conclusiones}

El b-learning es una modalidad efectiva en relación a los aprendizajes de conocimientos de los estudiantes, además propicia el desarrollo de habilidades de trabajo y aprendizaje autónomo. El profesor puede extender el trabajo del aula presencial y monitorear el trabajo de los estudiantes facilitando su rol en la retroalimentación 
a través de la automatización y entrega instantánea de los resultados.

Sobre experiencias en docencia universitaria que favorezcan la integración curricular de recursos informáticos y que innoven en estrategias evaluativas, protagonizadas por estudiantes en la construcción de sus aprendizajes, sigue siendo una tarea pendiente y escasa en docentes (Quesada et al., 2017), más aún, si estas tienen como destinatarios a futuros educadores en el contexto de su formación investigativa. Así, la estrategia de desarrollar habilidades evaluativas para el aprendizaje, de carácter instrumental, como lo es la elaboración de preguntas se asoció, solo en un caso de los tres estudiados, estadísticamente significativa con dominio de conocimientos. No obstante, Silvestri (2006), Macías y Maturano (2010), entre otros revelan que la creación de buenas preguntas sobre la lectura tiene un efecto positivo en el aprendizaje.

Con relación a la hipótesis investigativa del trabajo sobre la existencia de asociación entre las variables uso del aula virtual y dominio de conocimientos sobre conceptos clave de investigación educativa, se puede afirmar que en la mayoría de los casos estudiados esta asociación se dio, pero es baja.

Las implicaciones y aplicaciones de los resultados del estudio son sugerentes para la innovación de la práctica de la docencia en educación superior. En particular, esta se torna mucho más sinérgica si se integra en la formación de profesores, pues convergen pertinentes competencias de desarrollo profesional docente. Además, la facilidad de registrar, casi todo el proceso en Aula Virtual, permite evidenciar la efectividad de tal experiencia.

\section{Referencias bibliográficas}

Alexander, B., Ashford-Rowe, K., Barajas-Murphy, N., Dobbin, G., Knott, J., McCormack, M., Pomerantz, J., Seilhamer, R., \& Weber, N. (2019). EDUCAUSE Horizon Report: 2019 Higher Education Edition. Louisville: EDUCAUSE. https://bit.ly/2AA1IUN
Alfaro, M. (2000). Evaluación del aprendizaje. IUPEL. Alvares, I. (2008). La coevaluación como alternativa para mejorar la calidad del aprendizaje de los estudiantes universitarios: Valoración de una experiencia $[\mathrm{Co}$-assessment as an alternative to improve university students' learning quality: Evaluation of an experience]. Revista Interuniversitaria de Formación del Profesorado, 22(3), 127-140. http://bit.ly/32WfXfm

Arias, R., \& Venegas, H. (2013). Caracterización de los procesos de implementación y uso de las aulas virtuales en la Facultad de Educación. En J. Sánchez (Ed.), Nuevas ideas en Informática Educativa, Volumen 9 (pp. 343-352). Universidad de Chile.

Bahamondes, V., \& Ponce, M. (2012). Integración curricular de TIC en ámbito universitario: Claves de una experiencia constructivista. En J. Sánchez (Ed.), Nuevas ideas en Informática Educativa, 8 (pp. 292-296). Universidad de Chile.

Barberà, E. B., \& Badia, A. (2005). Hacia el aula virtual: Actividades de enseñanza y aprendizaje en la red [Towards virtual classroom: activities for teaching and learning process on the net]. Revista Iberoamericana de Educación, 36(9), 1-22. https://doi.org/10.35362/rie3692769

Bartolomé, A., García-Ruiz, R., \& Aguaded, I. (2018). Blended learning: Panorama y perspectivas [Blended learning: panorama y perspectivas]. RIED. Revista Iberoamericana de Educación a Distancia, 21(1), 33-56. http://doi.org/10.5944/ried.21.1.18842

Bausela, E. (2005). Ansiedad ante los exámenes: Evaluación e intervención psicopedagógica [Anxiety before tests: evaluation and psicopedagogic intervention]. Educere, 9(31), 553557. http://bit.ly/2obfQ0U

Boelens, R., Voet, M., \& De Wever, B. (2018). The design of blended learning in response to student diversity in higher education: Instructors' views and use of differentiated instruction in blended learning. Computers \& Education, 120, 197-212.

https://doi.org/10.1016/j.compedu.2018.02.009

Cardona, D., \& Sánchez, J. (2010). Indicadores básicos para evaluar el proceso de aprendizaje en estudiantes de educación a distancia en 
ambiente e-learning [Basic Indicators for Assessment the Learning Process in Students of Distance Education in Environment e-learning]. Formación Universitaria, 3(6), 15-32.

http://doi.org/10.4067/S0718-50062010000600004

Careaga, M., \& Fuentes, C. (2012). Contexto mixto de aprendizaje con apoyo de tutoría virtual para estudiantes de la carrera de pedagogía en educación media en lenguaje y comunicación. UCSC-Chile. En J. Sánchez (Ed.), Nuevas ideas en Informática Educativa, 8 (pp. 254-263). Universidad de Chile.

Carranza, M., \& Caldera, J. (2018). Percepción de los estudiantes sobre el aprendizaje significativo y estrategias de enseñanza en el Blended Learning [Perception of Students on Meaningful Learning and Teaching Strategies in Blended Learning]. REICE. Revista Iberoamericana sobre Calidad, Eficacia $y$ Cambio en Educación, 16(1), 73-88. https://doi.org/10.15366/reice2018.16.1.005

Castillo, V., Yahuita, J., \& Garabito R. (2006). Estrategias docentes para un aprendizaje significativo [Teacher strategies for a meaningful learning]. Revista Cuadernos del Hospital de Clínicas, 51(1), 96-101. http://bit.ly/331j5a4

Duart, J., \& Mengual, S. (2015). Transformaciones en la universidad hoy: Integración de modalidades formativas [Transformations in the University Today: Integrating formative models]. Revista Española de Educación Comparada, 26, 15-39.

https://doi.org/10.5944/reec.26.2015.14447

Dziuban, C., Graham, C., Moskal, P., Norberg, A., \& Sicilia, N. (2018). Blended learning: $T$

he new normal and emerging technologies. International Journal of Educational Technology in Higher Education, 15(1), 1-16. https://doi.org/10.1186/s41239-017-0087-5

Essam, R. (2010). Software Application for Computer Aided Vocabulary Learning in a Blended Learning Environment (Thesis of Master). The American University in Cairo. http://bit.ly/2oMRWZy

García-Beltrán, A., Martínez, R., Jaén, J., \& Tapia, S. (2016). La autoevaluación como actividad docente en entornos virtuales de aprendizaje/ enseñanza [Self-assessment in virtual tea- ching and learning environments]. Revista de Educación a Distancia, 50, 1-14.

http://doi.org/10.6018/red/50/14

González, M., Hernández, A., \& Hernández, A. (2007). El constructivismo en la evaluación de los aprendizajes del álgebra lineal [Constructivism in the evaluation of linear algebra]. Educere, 11(36), 123-135. http://bit.ly/32SEBh8

Halverson, L., \& Graham, C. (2019). Learner Engagement in Blended Learning Environments: A Conceptual Framework. Online Learning, 23(2),145-178. http://dx.doi.org/10.24059/olj.v23i2.1481

Hernández, R., Fernández, C., \& Baptista, P. (2012). Metodología de la investigación. McGraw Hill.

Kamii, C., \& López, P. (1982). Autonomy as an aim for education: Implications of Piaget's theory. Infancia y Aprendizaje, 5(18), 3-32. https://doi.org/10.1080/02103702.1982.10821934

Li, Z., Tsai, M., Tao, J., \& Lorentz, C. (2014). Switching to blended learning: The impact on students' academic performance. Journal of Nursing Education and Practice, 4(3), 245-251. https://doi.org/10.5430/jnep.v4n3p245

López, J. (2011). La importancia de formular buenas preguntas. http://bit.ly/2Vb34LM

López, M., Pérez, M., \& Rodríguez, L. (2013). Aplicación del aprendizaje combinado en contabilidad. Un análisis comparativo entre diferentes titulaciones universitarias [Aplicación del aprendizaje combinado en contabilidad. Un análisis comparativo entre diferentes titulaciones universitarias]. Revista de Educación, 360, 461-482. https://doi.org/10.4438/1988-592X-RE-2011-360-123

Luna, E., Ponce, S., Cordero, G., \& CisnerosCohernour, E. (2018). Marco para evaluar las condiciones institucionales de la enseñanza en línea [A Framework for Assessing Institutional Conditions of Online Teaching]. Revista Electrónica de Investigación Educativa, 20(2), 1-14. https://doi.org/10.24320/redie.2018.20.2.2072

Macías, A., \& Maturano, C. (2010). Evaluación de la comprensión a través de la formulación de preguntas por los estudiantes a partir de la lectura de un texto de física [Evaluation of comprehension through students' ques- 
tion asking when reading a text of physics]. Revista Signos, 43(74), 411-432.

http://doi.org/10.4067/S0718-09342010000500002

Manrique, L. (2004). El aprendizaje autónomo en la educación a distancia. http://bit.ly/2AGUCuo

Massié, A. (2010). El estudiante autónomo y autorregulado. http://bit.ly/2LIgNXA

McMillan, J.H., \& Schumacher, S. (2005). Investigación educativa. Pearson.

Ministerio de Educación de Chile MINEDUC (2012). Estándares orientadores para carreras de pedagogía en educación media. Santiago de Chile: MINEDUC.

Morón, F. (2015). La importancia de hacer buenas preguntas a nuestros alumnos de la ISO [The importance of appropriate questioning in ISO students]. Revista Arista Digital, 54, 1-12. http://bit.ly/2nfAtIV

Pacheco, S., Lisbeth, C., Brito, H., \& Carlos, R. (2014). Evaluación mediante el aula virtual en la enseñanza presencial de asignaturas de programación en ingeniería. Universidad de Carabobo, Facultad de Ingeniería.

Parra, E. (2008). Evaluación para los aprendizajes y la enseñanza. Ediciones UCSH.

Picciano, A., Dziuban, C., \& Graham, C. (2014). Blended learning: Research perspectives. Routledge.

Protopsaltis, S., \& Baum, S. (2019). Does Online Education Live Up to its Promise? A Look at the Evidence and Implications for Federal Policy. http://bit.ly/2IlCt9X

Quesada, V., Rodríguez, G., \& Ibarra, M.S. (2017). Planificación e innovación de la evaluación en educación superior: La perspectiva del profesorado [Design and Innovation of Assessment in Higher Education: Lecturers Perspective]. Revista de Investigación Educativa, 35(1), 53-70. https://doi.org/10.6018/rie.35.1.239261

Rienties, B., \& Toetenel, L. (2016). The impact of learning design on student behaviour, satisfaction and performance: A cross-institutional comparison across 15(2) modules. Computers in Human Behavior, 60, 333-341. https://doi.org/10.1016/j.chb.2016.02.074
Rojas, N., Pérez, F., Torres, I., \& Peláez, E. (2014). Las aulas virtuales: Una opción para el desarrollo de la Educación Médica [The virtual classroom: An option for the development of medical education]. EDUMECENTRO, 6(2), 231-247. http://bit.ly/353cNbN

Roza, J. C. da, Veiga, A. M. da R., \& Roza, M. P. (2019). Blended learning: Uma análise do conceito, cenário atual e tendências de pesquisa em teses e dissertações brasileiras [Blended learning an analysis of the concept, current scenario and research trends in brazilian thesis and dissertations]. ETD-Educação Temática Digital, 21(1), 202-221. https://doi.org/10.20396/etd.v21i1.8651638

Sajid, M., Laheji, A., Abothenain, F., Salam, Y., AlJayar, D., \& Obeidat, A. (2016). Can blended learning and the flipped classroom improve student learning and satisfaction in Saudi Arabia? International Journal of Medical Education, 7, 281-285. https://doi.org/10.5116/ijme.57a7.83d4

Sarramona, J. (2011). Autonomía y calidad de la educación. http://bit.ly/2oOlV3r

Silvestri, A. (2006). La formulación de preguntas para la comprensión de textos: Estudio experimental [Formulating questions to assess reading comprehension: Applied research]. Revista Signos, 39(62), 493-510. http://doi.org/10.4067/S0718-09342006000300008 UCSH (2016). Campus Virtual. http://bit.ly/32Yw8ce Vaughan, N. (2010). Designing for a blended community of inquiry. En T. Joutsenvirta y L. Myyry (2010) (Eds.), Blended learning in Finland (pp. 11-29). Helsinki: Faculty of Social Sciences at the University of Helsinki.

Vivel-Bua,M., Fernández, S., \& Lado-Sestayo, R. (2015). Innovación docente con One Minute Paper, ¿afecta el rendimiento escolar? [Educational Innovation through One Minute Paper: Does it Affect Academic Performance?]. Revista Electrónica de Investigación Educativa, 17(2), 48-61. http://bit.ly/30J9ovl 